\title{
Molecular Communication through Gap Junction Channels: System Design, Experiments and Modeling
}

\author{
Tadashi Nakano, Tatsuya Suda \\ Department of Computer Science \\ Donald Bren School of Information and Computer Sciences \\ University of California, Irvine \\ \{tnakano, suda\}@ics.uci.edu \\ Takako Koujin, Tokuko Haraguchi, Yasushi Hiraoka \\ Kobe Advanced ICT Research Center \\ National Institute of Information and Communications Technology \\ \{koujin, tokuko,yasushi\}@nict.go.jp
}

\begin{abstract}
Molecular communication is engineered biological communication that allows nanomachines to communicate through chemical signals. Nanomachines are small scale biological devices that either exist in nature or are artificially engineered from biological materials, and that perform simple functions such as sensing, processing, and actuation. As nanomachines are too small and simple to communicate through a traditional communication means (e.g. electromagnetic waves), molecular communication provides a mechanism for nanomachines to communicate by propagating molecules that represent information. In this paper, we propose to explore biological cells for engineering a molecular communication system. Its system characteristics and key networking services are first discussed, and then our current status of experimental and modeling studies is briefly reported.
\end{abstract}

\section{Keywords}

Synthetic biological systems, molecular communication, gap junction channels, calcium signaling

\section{INTRODUCTION}

Communication plays a critical role in a broad range of nano and microscale applications from molecular computing, biochemical sensing to nanomedicine. Communication provides a means by which nanomachines (e.g., engineered organisms or biological devices) coordinately perform tasks that cannot be accomplished by a single nanomachine. For example, nanomachines that can function as basic logic gates [33] may perform distributed computing through communication; medical nanomachines [9] with communication capabilities may perform coordinated monitoring of human health.

Permission to make digital or hard copies of all or part of this work for personal or classroom use is granted without fee provided that copies are not made or distributed for profit or commercial advantage and that copies bear this notice and the full citation on the first page. To copy otherwise, or republish, to post on servers or to redistribute to lists, requires prior specific permission and/or a fee.

Bionetics'07, December 10-13, 2007, Budapest, Hungary.

Copyright 2007 ICST 978-963-9799-11-0

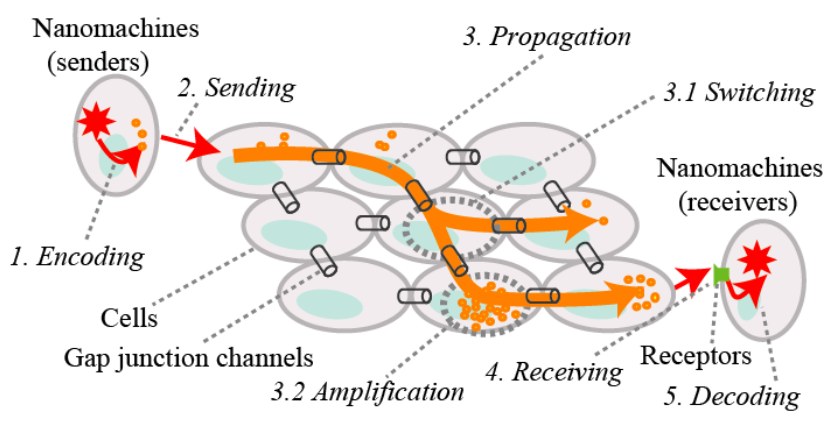

Figure 1: A design of molecular communication system

It is known that living cells in various tissues and organisms utilize numerous networking mechanisms to establish cell-cell communications. Existing cell-cell communication mechanisms may be thus applicable to engineering molecular communication systems (i.e., synthetic biological communication systems) [12, $21,22]$. In addition, current molecular engineering technologies may allow modification of existing cell-cell communication mechanisms to provide advanced networking functionality such as that used in today's computer networking.

Figure 1 depicts a design of a biological cell-based molecular communication system inspired by cell-cell communications through gap junction channels. Nanomachines in this figure are engineered organisms or biological devices whose behavior is programmed to achieve application specific goals, and chemically communicate over a cell-cell communication medium. To support molecular communication between nanomachines, the communication medium may provide various networking mechanisms and services such as signal amplification and switching.

To illustrate how communication is performed in the system described in Figure 1, consider the following example. The sender senses some toxic chemical substances in the environment and synthesizes signal molecules that can initiate cell-cell signaling (encoding). The sender then emits the signal molecules (sending), which trigger cell-cell signaling. The signal molecules propagate cell-cell through gap junction channels (propagation). During propagation, signal molecules may be directed toward target 
receivers (switching) or amplified for longer distance propagation (amplification). The receiver reacts to nearby cells that start receiving the signals (receiving), and initiates biochemical processes such as secreting neutralizing chemicals into the environment (decoding).

The rest of the paper is organized as follows. Section 2 describes general characteristics and features of biological cell-based molecular communication systems. Section 3 discusses a possible design of the biological cell-based molecular communication system based on gap junctional communication. Section 4 demonstrates a simple molecular communication medium experimentally. Section 5 describes a modeling study performed to understand communication related characteristics of the molecular communication and Section 5 concludes this paper.

\section{SYSTEM CHARACTERISTICS}

Biological cell-based molecular communication systems may exhibit several distinct features from silicon based counterparts (computer network systems). As will be described in the following, such features may be explored to design and develop new Bio-ICT (Information and Communications Technology) applications while at the same time such features may limit the applicability of molecular communication systems.

Small scale, limited range and slow speed communication: The system size may vary from um to $\mathrm{cm}$ depending on cell types (10 30um for eukaryotic cells) and a cellular structure (cm or more) formed to implement a system. The communication range between system components (cells) is strictly limited and communication speed is extremely slow compared to existing telecommunications (speed of light). For example, the longest range and fastest communication possible would be when neural signaling is used as a communication channel, in which case electro-chemical signals (action potentials) may propagate up to several meters at $100 \mathrm{~m} / \mathrm{sec}$. In other cases, where $\mathrm{Ca}^{2+}$ waves of astrocytes are used, communication is much slower and $20 \mathrm{um} / \mathrm{sec}$ within a cm range [26].

Functional complexity: An advantageous feature of using cells as a system component is achievable functional complexity. A cell is a highly functional and integrated component with information processing capabilities. A cell has a number of sensors (e.g., receptors to sense the environment), logic circuits (e.g., complex signal transduction pathways), memory for storing information, and actuators that can generate motion. A functional density of a bacterial species, Escherichia coli, is estimated in [6], which states that a cell stores a 4.6 million basepair chromosome in a $2 \mathrm{um}^{2}$ area, which is equivalent to a 9.2-megabit memory that encodes a number of functional polypeptides. Functional complexity may help a design of highly compacted engineered systems including NEMS/MEMS (nano- and microelectromechanical systems), lab-on-a-chips and u-TAS (MicroTotal Analysis Systems).

Biocompatibility: Another advantageous feature of using cells is biocompatibility. Cells can interact directly with other cells, tissues and organs through receiving, interpreting, synthesizing and releasing molecules, and thus biological cell-based molecular communication systems may be useful in medical domains (e.g., implantable devices, cell-based biosensors, body sensor networks [31]), where interactions with a human body are necessary. Also, molecular communication may be indispensable for communication between soft nanomachines that are composed of biological materials (e.g., molecular computing devices) and that are not capable of transmitting and receiving traditional communication signals (electromagnetic waves).

Chemical energy, energy efficiency, and low heat dissipation: Biological cell-based molecular communication systems operate with chemical energy (e.g., ATP), unlike silicon devices that require electric batteries. Chemical energy may be possibly supplied by the environment where molecular communication systems are situated. For example, molecular communication systems deployed in a human body may harvest energy (e.g., glucose) from the human body, requiring no external energy sources. Also, biological cell-based molecular communication systems may be energy efficient with low heat dissipation as cellular components are energy efficient. For example, an F1ATP motor converts ATP energy to mechanical work at nearly 100 percent energy efficiency [15].

Self-assembly: Self-assembly is a possible property of biological cell-based molecular communication systems. Cells can divide and grow to assemble into a larger structure (organs). This selfassembly property may be exploited in system design of molecular communication systems, enabling a bottom up system fabrication and deployment. In addition, self-assembling molecular communication systems are highly fault-tolerant as damaged parts of a system may be recovered through division and growth of nearby cells.

Probabilistic behavior: Biological cell-based molecular communication systems will be placed in an aqueous environment where thermal noise and other environmental factors may affect the system behavior. For example, signal molecules may randomly propagate based on Brownian motion. Signal molecules may also be broken down or degraded during propagation, introducing probabilistic and unpredictable behavior. For molecular communication, such probabilistic aspects may be overcome by relying on a large number of molecules for communication (ensemble averaging). On the other hand, thermal noise may be utilized to enhance a signal-to-noise ratio with stochastic resonance as is likely performed in some organisms (e.g., amoeba's chemotaxis, neural information processing).

Robustness and fragility: Cells or cellular systems exhibit some degree of robustness against internal and external perturbations [16]. Cells have evolutionary acquired control mechanisms (feedback mechanisms) to achieve robustness and maintain stability (e.g., homeostasis). At the same time, cells are extremely fragile to various factors such as temperature and $\mathrm{pH}$ changes that can destruct system behavior easily. Biological cell-based molecular communication systems may inherit such features of robustness and fragility.

Safely issues - Although biological cell-based systems have a number of features that silicon devices may not have, safely issues definitely arise, especially when used for medical and environmental applications (e.g., that may introduce new infectious viruses). Potential risks must be assessed in order to advance this technology field.

\section{SYSTEM DESIGN}

Synthetic biological communication systems have been designed and experimentally demonstrated in synthetic biology $[2,32]$. These systems use unguided communication media in which 
bacterial cells diffuse paracrine type signals in the extracellular environment. Another type of communication is possible by using guided communication media such as gap junction channels that can guide diffusion of signal molecules.

The biological cell-based molecular communication system presented in this paper is designed based on the latter type of communication media. The present system specifically relies on diffusion of signal molecules through gap junction channels (Figure 1). The following firstly provides a brief introduction to gap junction channels before proceeding to the system design.

\subsection{Gap Junction Channels}

Gap junction channels [26] are physical channels formed between two adjacent cells, connecting the cytoplasm of the two cells (Figure 2). A gap junction channel consists of two apposed hexamers of connexin proteins around a central pore. There are over 20 connexins reported, and different connexins can constitute gap junction channels with different properties in terms of permeability and selectivity of molecules [24]. Gap junction channels normally allow the passage of small molecules (<1000Da) (e.g., ions, $\mathrm{IP}_{3}$, cyclic AMP) between cells. However, gap junction channels can vary the permeability and selectivity in response to various internal and external factors including cytosolic $\mathrm{Ca}^{2+}$, membrane potential, connexin phosphorylation, electromagnetic fields, temperature and $\mathrm{pH}$ in the environment.

Gap junction channels play an important role in propagating signal molecules including $\mathrm{Ca}^{2+}$ signals (known as intercellular $\mathrm{Ca}^{2+}$ wave propagation). For example, glial cells have $30-150 \mathrm{nM}$ of cytosolic $\mathrm{Ca}^{2+}$ concentration (denoted as $\left[\mathrm{Ca}^{2+}\right]_{\mathrm{i}}$ ) at rest, and upon stimulated, $\left[\mathrm{Ca}^{2+}\right]_{\mathrm{i}}$ increases up to hundreds of $\mathrm{nM}$ or several $\mu \mathrm{M}$ within milliseconds while propagating the increase from cell to cell [7].

There are various models that explain intercellular $\mathrm{Ca}^{2+}$ wave propagation. In one model, stimulated cells produce $\mathrm{IP}_{3}\left(\mathrm{Ca}^{2+}\right.$ mobilizing molecules) that diffuses through gap junction channels from cell to cell, stimulates $\mathrm{Ca}^{2+}$ stores at each cell, and triggers $\mathrm{Ca}^{2+}$ release from the stores at each cell. $\mathrm{As} \mathrm{IP}_{3}$ diffuses cell-cell, intercellular $\mathrm{Ca}^{2+}$ waves propagate. In the other model, stimulated cells produce ATP that diffuses through gap junction hemichannels to the extracellular environment. Released ATP reacts with membrane receptors at each cell, and each cell produces $\mathrm{Ca}^{2+}$ mobilizing molecules (e.g., $\mathrm{IP}_{3}$ ) and triggers $\mathrm{Ca}^{2+}$ release from $\mathrm{Ca}^{2+}$ stores. As ATP diffuses in the extracellular environment, intercellular $\mathrm{Ca}^{2+}$ waves propagate. In some other models, $\mathrm{Ca}^{2+}$ itself propagates through gap junction channels and causes $\mathrm{Ca}^{2+}$ release at each cell (calcium induced calcium release), thereby generating self-regenerative $\mathrm{Ca}^{2+}$ waves.

Intercellular $\mathrm{Ca}^{2+}$ waves can potentially propagate biological information such as cell death [18] and growth [28]. More complex information may be propagated because $\mathrm{Ca}^{2+}$ signals are used as a universal second messenger. It is known that temporal and spatial dynamics of cytosolic $\mathrm{Ca}^{2+}$ (termed as $\mathrm{Ca}^{2+}$ spikes/oscillations) are modulated to encode various cellular activities, and the $\mathrm{Ca}^{2+}$ dynamics is decoded by $\mathrm{Ca}^{2+}$ sensitive molecules that trigger various cellular responses $[4,5]$.
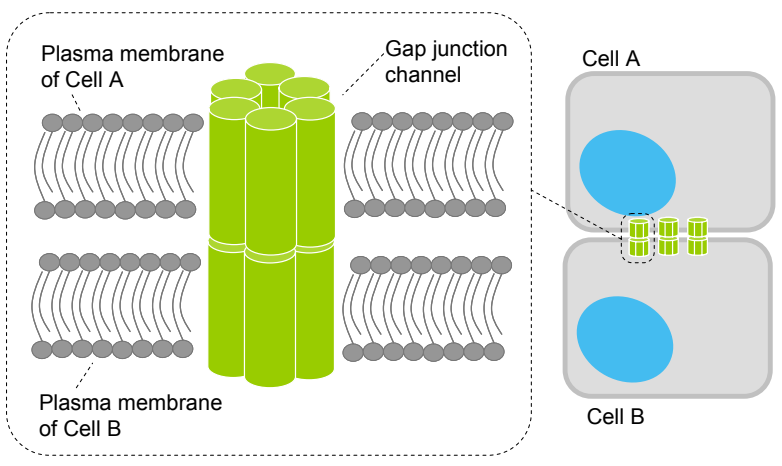

Figure 2: Gap junction channels. Gap junctions are cellcell communication channels formed between two adjacent cells, allowing small molecules such as ions and metabolites to diffuse between the cytoplasms of the two cells.

\subsection{Molecular Communication through Gap Junction Channels}

A generic molecular communication system [21] is composed of signal molecules that carry information to be transmitted, senders that transmit signal molecules, receivers that receive signal molecules, and the environment in which signal molecules propagate from senders to receivers. In the present molecular communication system, $\mathrm{Ca}^{2+}$ signals are used as signal molecules, and a gap junctionally connected cells as the environment. Senders and receivers are application specific and remain as ge abstract entities.

A generic molecular communication [21] is performed between senders and receivers through the five key communication processes: encoding (process by which senders translate biochemical reactions into signal molecules), sending (process by which senders release signal molecules into the environment), propagation (process by which signal molecules move through the environment from senders to receivers), receiving (process by which receivers detect signal molecules propagating in the environment), and decoding (process by which receivers, after detecting signal molecules, decodes the molecules into biochemical reactions.)

Relative to the generic communication processes, molecular communication of the present molecular communication system (Figure 1) can be described as follows.

1. Encoding: $\mathrm{Ca}^{2+}$ spikes/oscillations are used to represent information. Encoding is the process by which senders select the properties of $\mathrm{Ca}^{2+}$ dynamics. This includes choices of $\mathrm{Ca}^{2+}$ mobilizing molecules and the amount of the molecules to release (e.g., agonistic substances in certain amounts).

2. Sending: Sending is the process of releasing $\mathrm{Ca}^{2+}$ mobilizing molecules in the manner decided in the encoding process. $\mathrm{Ca}^{2+}$ mobilizing molecules may be released constantly, in a burst-like manner or in an even more complex manner. Released $\mathrm{Ca}^{2+}$ mobilizing molecules react with membrane receptors of neighboring cells, activating downstream signaling pathways that lead to increase of $\left[\mathrm{Ca}^{2+}\right]_{\mathrm{i}}$ in the cells. 
3. Propagation: The increase of $\left[\mathrm{Ca}^{2+}\right]_{\mathrm{i}}$ propagates from cell to cell through gap junction channels. Cells may perform amplification of cytosolic $\mathrm{Ca}^{2+}$ to increase the propagation distance (calcium induced calcium release). Cells may also perform switching by opening and closing gap junctions, so that $\mathrm{Ca}^{2+}$ waves propagate preferentially toward target receivers.

4. Receiving: In receiving, receivers detect cellular responses of neighboring cells that have received $\mathrm{Ca}^{2+}$ waves. For example, neighboring cells experiencing changes in its $\left[\mathrm{Ca}^{2+}\right]_{\mathrm{i}}$ may release molecules into the extracellular environment, and nearby receivers capture the released molecules using receptors.

5. Decoding: Receivers invoke application specific responses corresponding to molecules captured.

\subsection{Design Detail}

Some more detail of the system design follows in comparison to computer networks design [29]. Looking into computer networks design may help identify necessary mechanisms and functionality for molecular communication. For example, typical physical layer issues such as types of communication media, modulation techniques, signal amplification and noise handling may also be important for molecular communication. In addition, mechanisms and functionality from upper layers of computer networks may also be useful for molecular communication, including synchronization, error detection/ correction, filtering, switching, addressing, flow control, and so on. A possible design of some of the mechanisms and functionality is discussed below.

Communication media: There are two common types of communication media by which cells communicate with each other. In one type, similar to guided media of computer networks (e.g., coax cables, fiber optics), cells establish direct contact through gap junction channels, restricting diffusion of signal molecules (e.g., $\mathrm{Ca}^{2+}, \mathrm{IP}_{3}$ ) within cells connected through gap junction channels. In the other type, similar to unguided media of computer networks (e.g., wireless), cells diffuse signal molecules (e.g., ATP, cyclic AMP) in the extracellular environment, and nearby cells respond to the molecules in the environment (paracrine signaling). Accordingly, the present molecular communication system can also have the two types of communication media.

Modulation techniques: Interestingly, cells adopt various modulation schemes to represent data (or messages), similar to modulation technique used in computer networks or radio broadcast (FM/AM). For example, in calcium signaling, messages are represented in complex temporal dynamics of calcium concentrations, referred to as AM and FM of calcium signaling [3]. Similarly, some cells can secrete molecules in the extracellular environment in a complex manner (e.g., in an oscillatory manner) to represent some messages. Accordingly, the present molecular communication system may be able to implement or utilize their built-in modulation techniques.

Signal attenuation and noise handling: Cells use various feedback mechanisms; a positive feedback to amplify signals and a negative feedback to reduce the impact of noise. For example, CICR (Calcium Induced Calcium Release) is a mechanism to amplify calcium signals. It is recently found that ATP signals are also amplified in some cell types termed as ATP induced ATP
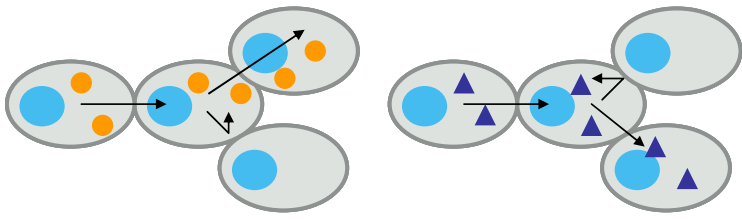

Figure 3: Signal filtering. The four cells together perform signal filtering as follows. One type of incoming signal molecules represented as circles is more permeable to gap junction channels as indicated in the left figure. Similarly, the other type of incoming signals represented as triangles is as indicated in the right figure.

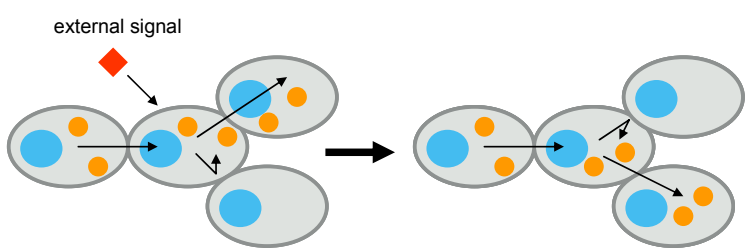

Figure 4: Signal switching. The external signal represented by rectangle modifies permeability of gap junction channels, dynamically changing characteristics of signal propagation.

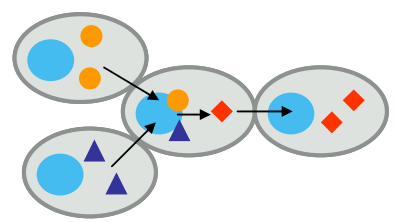

Figure 5: Signal aggregation. Two incoming signal molecules (represented as circles and triangles) that are not permeable to the rightmost cell react in the centered cell to produce signal molecules (represented as rectangles) that are permeable to the rightmost cell.

release [1]. For noise handling, there is theoretical work that thermal noise is utilized by cells to increase a signal-to-noise ration through SR (Stochastic Resonance) [19]. These mechanisms may also be incorporated into the present molecular communication system.

Filtering: Gap junction channels can have different selectivity and permeability, and this property can be used to implement filters for molecular communication, similar to packet filtering used in computer networks. Figure 3 illustrates a simple example of filtering using gap junction channels. Gap junction channels formed between cells can have different permeability and thus signals molecules may preferentially propagate (see either the left four cells or right four cells in Figure 3). In addition, gap junction channels can have different selectively, allowing different signal molecules to diffuse differently (compare the propagation on the left and right).

Switching: The selectivity and permeability of gap junction channels can vary in response to various factors (e.g., phosphorylation of connexin proteins), and this dynamic property can be used to implement something like dynamic switching of computer networks. Figure 4 illustrates a design of dynamic 

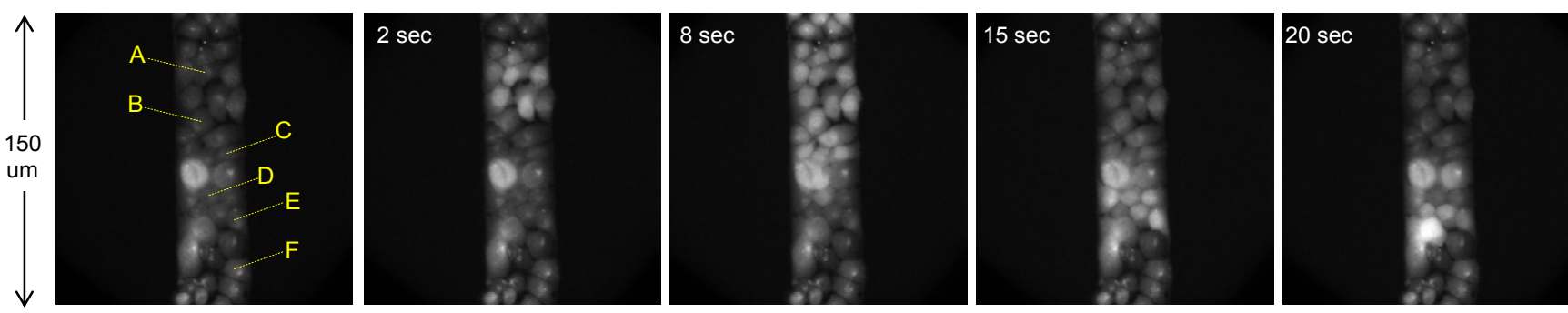

Figure 6: Cell wire that propagates $\mathrm{Ca}^{2+}$ waves over $\mathrm{HeLa} \mathrm{Cx} 43$ cells

switching, where the permeability to signal molecules are changed by external signals.

Signal aggregation: The selectivity and permeability of gap junction can be further exploited to perform more complex networking functionality such as signal aggregation. Figure 5 shows an example of this kind. One type of signal flows into the centered cell, but this signal is not permeable to the rightmost cell, therefore no further propagation. However, once the other type of signal comes in the centered cell, chemical reactions of the two types of signals produce signals that are permeable to the rightmost cell, therefore propagating signals. This can be viewed as signal aggregation.

\section{Cell Wires}

In this section, we experimentally demonstrate a molecular communication medium, cell wires that propagate $\mathrm{Ca}^{2+}$ waves along a line of gap junction transfected HeLa cells [24]. Briefly, a microplatform for cell-patterning was first established by utilizing surface chemistry, and HeLa Cx43 cells were patterned onto the platform. Flash-photolysis of caged-ATP, caged-Ca ${ }^{2+}$, caged-IP ${ }_{3}$, or mechanical stimulation was then used to initiate intercellular $\mathrm{Ca}^{2+}$ waves that propagated along a line of cells. For more detail of experimental procedures and results, see [23].

Figure 6 shows a cell wire that propagated intercellular $\mathrm{Ca}^{2+}$ waves along a line of patterned HeLa Cx43 cells in response to photo-release of ATP. In this experiment, cell A was flashed, which increased its cytosolic $\mathrm{Ca}^{2+}$. The increased $\mathrm{Ca}^{2+}$ level then propagated along the cell line at a rate of about $5 \mu \mathrm{m} / \mathrm{sec}$, and reached the cell that was 10 cells away from the flashed cell. Figure 7 shows the fluorescence intensity of cells A-F during the course of fluorescence measurements. The experimental results shown in Figures 6 and 7 were reproducible for a number of times $(\mathrm{n} \geq 10)$, and similar results were obtained from the repeated experiments. The $\mathrm{Ca}^{2+}$ wave propagation in this experimental setup was presumably facilitated by gap-junctional diffusion of messenger molecules (e.g., $\mathrm{Ca}^{2+}$ itself, $\mathrm{IP}_{3}$ ) in addition to extracellular diffusion of photo-released ATP. Further experiments are currently being conducted to identify molecular mechanisms associated with this $\mathrm{Ca}^{2+}$ wave propagation.

HeLa Cx43 cells successfully propagated $\mathrm{Ca}^{2+}$ signals cell-to-cell suggesting the feasibility of using $\mathrm{HeLa} \mathrm{Cx} 43$ cells for implementing biological-cell based molecular communication media. The achieved distance and speed of $\mathrm{Ca}^{2+}$ waves, although within the commonly observed range [26] (a few um to 500 um or more, at a few um to $100 \mathrm{um} / \mathrm{sec}$ ), may be unsatisfactorily for

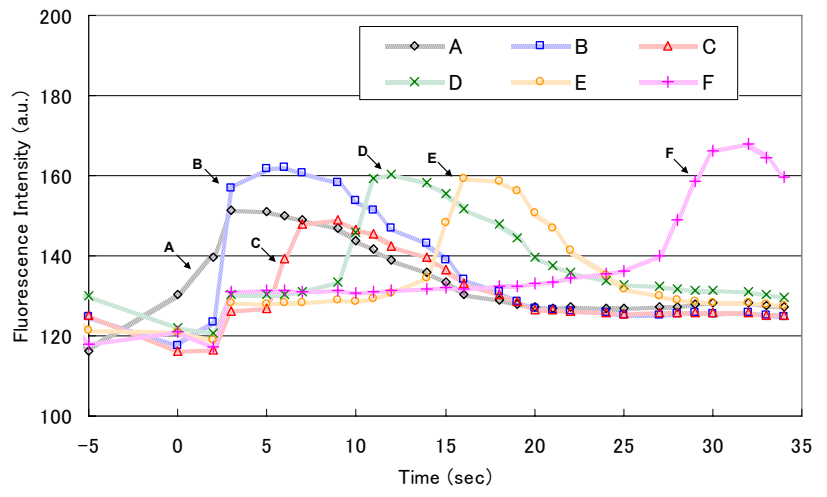

Figure 7: Fluorescence intensity of cells A-F

particular needs of applications. Increasing the possible distance and speed of communication may benefit a wider range of applications. Gap junctional connectivity is one of the factors influencing the property of communication, and how gap junctional connectivity affects the speed and distance of propagating $\mathrm{Ca}^{2+}$ waves is examined in the following modeling study.

\section{Modeling Study}

A modeling study is conducted to investigate communication related characteristics of molecular communication through gap junction channels. A mathematical model is used to examine the impact of gap junctional coupling on the properties of intercellular $\mathrm{Ca}^{2+}$ waves (e.g., possible distance and speed of traveling waves.)

Figure 8 schematically illustrates the model used in this study. The model is based on a classical model of calcium oscillation of a single cell [10], and a minor extension is made to simulate intercellular $\mathrm{Ca}^{2+}$ waves over an array of cells. Briefly, the $\mathrm{Ca}^{2+}$ concentration of the cytosol at cell $i$ is represented as $Z_{i}$ and that of the calcium store is $Y_{i}(i=0,1,2, \cdots, n-1)$. More specifically, the time evolution of $Z_{i}$ and $Y_{i}$ is described as follows: 


$$
\begin{aligned}
& \frac{d Z_{i}}{d t}=v_{0}+i n_{i}-v_{2 i}+v_{3 i}+k_{f} Y_{i}-k Z_{i}+P\left(Z_{i-1}-2 Z_{i}+Z_{i+1}\right) \\
& \frac{d Y_{i}}{d t}=v_{2 i}-v_{3 i}-k_{f} Y_{i} \\
& v_{2 i}=V_{M 2} \frac{Z_{i}^{n}}{K_{2}^{n}+Z_{i}^{n}} \\
& v_{3 i}=V_{M 3} \frac{Y_{i}^{m}}{K_{R^{m}}+Y_{i}^{m}} \frac{Z_{i}^{p}}{K_{A^{p}}^{p}+Z_{i}^{p}}
\end{aligned}
$$

Most of the constants and notations in the above equations are barrowed from [10]; $v_{0}$ represents $\mathrm{Ca}^{2+}$ influx from the extracellular environment to the cytosol; $i n_{i}$ a stimuli applied to cell $i$ (replaced with $v_{1}{ }^{*} B$ in [10]); $v_{2 i}$ and $v_{3 i}$ respectively $\mathrm{Ca}^{2+}$ uptake to and release from the calcium store; $k_{f} Y_{i}$ leaky $\mathrm{Ca}^{2+}$ transport from the calcium store to the cytosol; $k Z_{i} \mathrm{Ca}^{2+}$ transport from the cytosol to the extracellular environment. $P$ (coupling parameter) is added to the original model to represent the degree of gap junctional connectivity between two adjacent cells. $v_{2 i}$ and $v_{3 i}$ are further described in the last two equations using rate constants, threshold constants and Hill coefficients $\left(V_{M 2}, K_{2}, n\right.$, $\left.V_{M 3}, K_{R}, m, K_{A}, p\right)$.

In the simulation, cell 0 is stimulated at time 0 for a short period of time, $d$. Therefore, $i n_{i}=v_{1}{ }^{*} B$ for $i=0$ and for time 0 to $d$; and $i n_{i}=0$, otherwise. Two sets of parameter values are prepared as follows, and communication related characteristics are examined.

- Parameter set1: $v_{0}=1(\mathrm{uM} / \mathrm{s}), v l=7.3(\mathrm{um} / \mathrm{s}), B=0.01$, $k=3.5(1 / \mathrm{s}), k_{f}=1(1 / \mathrm{s}), V_{M 2}=50(\mathrm{uM} / \mathrm{s}), V_{M 3}=500(\mathrm{uM} / \mathrm{s})$, $K_{2}=1$ (uM), $K_{R}=2(\mathrm{uM}), K_{A}=0.9(\mathrm{uM}), m=n=2, p=4, d=0.5$ (sec) and $n=50$. ( $P$ is varied.)

- Parameter set2: $V_{M 2}=60(\mathrm{uM} / \mathrm{s}), V_{M 3}=400(\mathrm{uM} / \mathrm{s})$ and other values are the same as parameter set 1 .

The model equations are numerically solved using the Euler's method, and the speed and distance of traveling waves are examined as a function of coupling parameter $(P)$. Figures 9 and 10 plot the results obtained from parameter sets 1 and 2, respectively.

In both cases, the coupling parameter $(P)$ has a similar impact on the distance of waves. If $P$ is very small $(\sim 0.15$ for parameter set 1 and $\sim 0.12$ for parameter set 2), intercellular $\mathrm{Ca}^{2+}$ waves are either not generated or fail to travel (i.e., travel only a couple of cells). If $P$ reaches a certain value $(0.16$ for parameter set 1 and 0.13 for parameter set 2), intercellular $\mathrm{Ca}^{2+}$ waves travel up to the last cell of the array (50th cell). As will be shown in Figure 12, the waves become regenerative in this case. If $P$ is further increased (1.2 for parameter set 1 and 0.3 for parameter set 2), intercellular $\mathrm{Ca}^{2+}$ waves are not generated at all. This is because the initial stimulating $\mathrm{Ca}^{2+}$ at cell 0 (i.e., $i n_{0}$ ) diffuses so first between cells that cell 0 and other cells fail to trigger $\mathrm{Ca}^{2+}$ release from the calcium store to increase cytosolic $\mathrm{Ca}^{2+}$ (thus, no first spike appearing at cell 0 ).

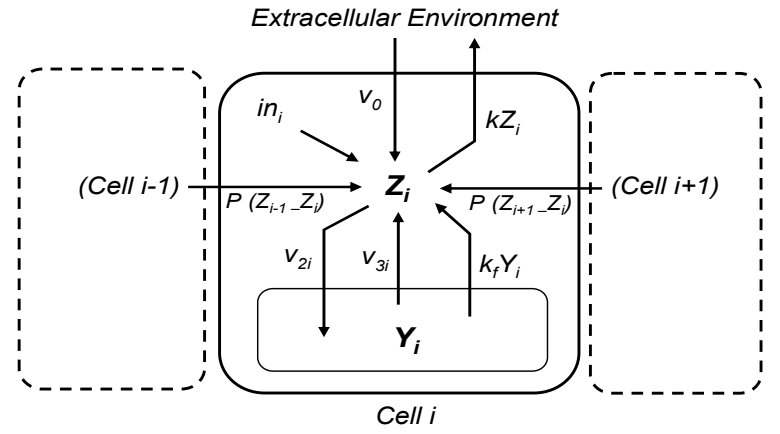

Figure 8: Model of intercellular $\mathrm{Ca}^{2+}$ wave propagation

Also, in both cases, the coupling parameter $(P)$ has a similar impact on the speed of waves. Larger $P$ values increase the speed of intercellular $\mathrm{Ca}^{2+}$ waves traveling. Note in Figures 9 and 10 the traveling speed is measured only when waves became regenerative and propagated to the 50th cell.

Figures 11 and 12 depict two representative cases where intercellular $\mathrm{Ca}^{2+}$ waves fail to be regenerative (from parameter set1, $\mathrm{P}=0.15$ ), and become regenerative (from parameter set1, $\mathrm{P}=0.16$ ). In the case of propagation failure, the wave peak attenuates while traveling (Figure 11), while in the case of successful propagation the wave peak stays the same and propagates up to the last cell of the array (Figure 12). Note in Figure 12 that the peak of cell 0 is different from that of others. This is because cell 0 responds to $i n_{i}$ (the initial stimuli) to generate a spike, while cell i $(i \neq 0)$ basically responds to $P\left(Z_{i-I}-Z_{i}\right)$ $\left(\mathrm{Ca}^{2+}\right.$ influx from cell i-1) to generate a spike.

The modeling study described in this section examines only one aspect of molecular commutation using a simple model, and a further study needs to be performed to obtain a deeper understanding of the communication properties. Remaining work on modeling studies includes (1) experimentally validating the model and demonstrating the impact of gap junctional permeability, (2) considering stochastic and spatial aspects in intracellular calcium wave propagation $[11,17]$, (3) use of a more complex model such as [13, 27] or mathematical approximation such as [14] to identify key communication related characteristics, and (4) quantifying channel capacities from an information theoretic point of view as studied in other systems [8,25,30] (our work on this aspect is partly presented in [20]).

\section{CONCLUDING REMARKS}

This paper presented system design, experiments and modeling of a biological cell based molecular communication system. Our study on the molecular communication system has just begun, and there remain many research issues that need to be addressed in all the three aspects of the system design, experiments and modeling.

\section{ACKNOWLEDGMENTS}

This research is supported by the NICT (the National Institute of Communication Technology, Japan), by the NSF through grants ANI-0083074, ANI-9903427 and ANI-0508506, by DARPA 


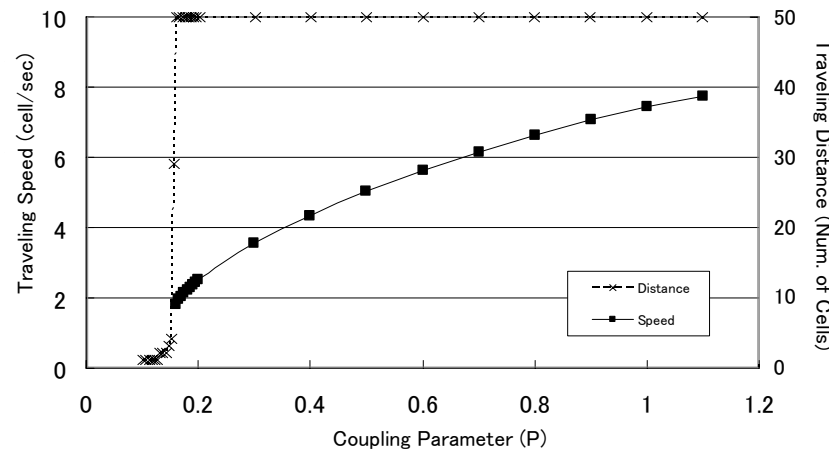

Figure 9: Traveling distance and speed from parameter set 1

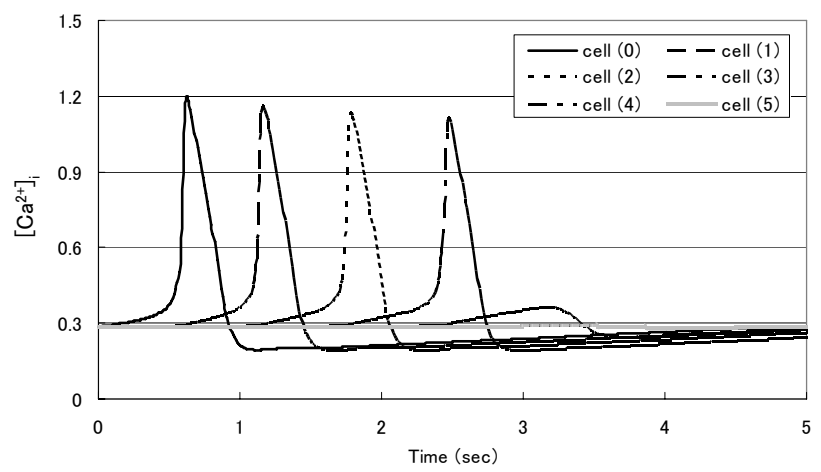

Figure 11: Propagation failure

(parameter set 1 and $\mathrm{P}=0.15$ )

through grant MDA972-99-1-0007, by AFOSR through grant MURI F49620-00-1-0330, and by grants from the California MICRO and CoRe programs, Hitachi, Hitachi America, Hitachi CRL, Hitachi SDL, DENSO IT Laboratory, DENSO International America LA Laboratories, NTT Docomo and Novell.

\section{REFERENCES}

[1] Anderson, C. M., J. P. Bergher, R. A. Swanson, "ATPinduced ATP release from astrocytes," J Neurochem (2004) 88: $246-56$

[2] Basu, S., Y. Gerchman, C. H. Collins, F. H. Arnold, and R. Weiss, "A synthetic multicellular system for programmed pattern formation," Nature. Vol. 434, 1130-1134, 2005.

[3] Berridge, M. J., "The AM and FM of calcium signaling," Nature, 386:759-780, 1997.

[4] Berridge, M. J., M. D. Bootman and P. Lipp, "Calcium - a life and death signal," Nature 395, 645-648, 1998.

[5] Berridge, M. J., P. Lipp and M. D. Bootman, "The versatility and universality of calcium signaling," Nature Reviews, Molecular Cell Biology, Vol. 1, pp. 11-21, 2000.

[6] Doktycz, M. J. and M. L. Simpson, "Nano-enabled synthetic biology," Molecular Systems Biology 3:125, 2007.

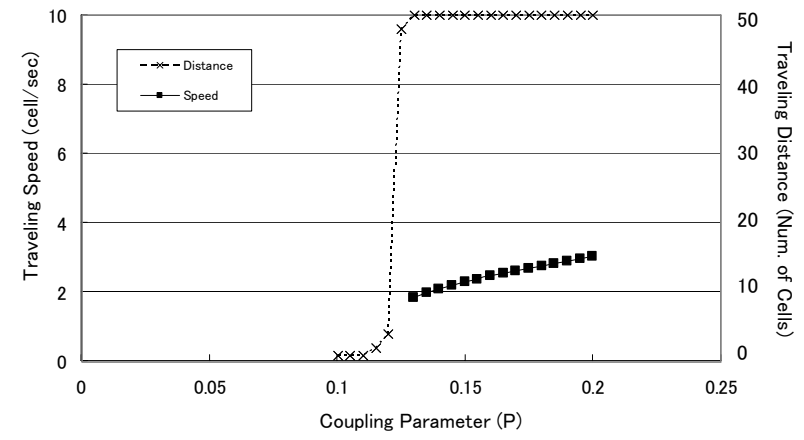

Figure 10: Traveling distance and speed from parameter set 2

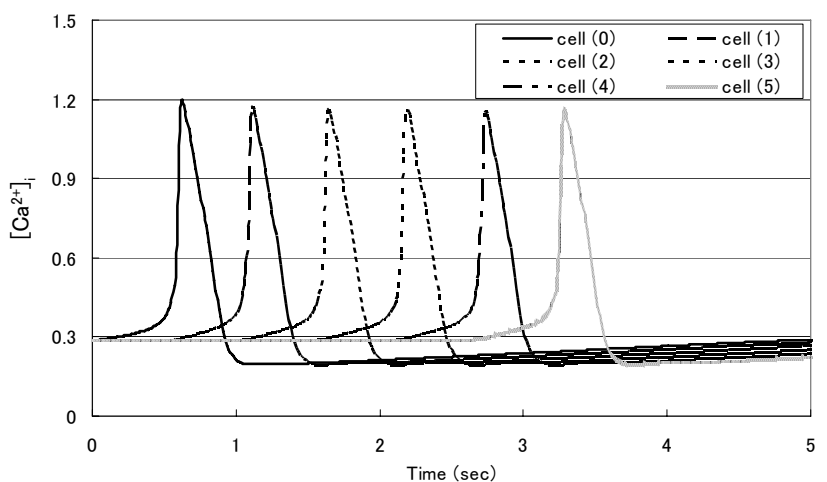

Figure 12: Suuccessful propagation

(parameter set 1 and $\mathrm{P}=0.16$ )

[7] Deitmer, J.W., A.J. Verkhratsky, C. Lohr, "Calcium signaling in glial cells," Cell Calcium, 24(5-6):405-16, 1998.

[8] Eckford, A., "Nanoscale communication with Brownian motion," in Proc. 41st Annual Conference on Information Sciences and Systems (CISS 2007), 2007.

[9] Freitas Jr., R.A., Nanomedicine, vol. I: Basic Capabilities, Landes Bioscience, 1999.

[10] Goldbetter, A., G Dupont and MJ Berridge, Minimal model for signal-Induced $\mathrm{Ca}^{2+}$ oscillations and for Their frequency encoding through protein phosphorylation," Proceedings of the National Academy of Sciences, vol. 87, 1461-1465, 1990.

[11] Gracheva, M. E., R. Toral, and J. D. Gunton, "Stochastic effects in intercellular calcium spiking in hepatocytes," Journal of Theoretical Biology, vol. 212, pp. 111-125, 2001.

[12] Hiyama, S., Y. Moritani, T. Suda, R. Egashira, A. Enomoto, M. Moore and T. Nakano, "Molecular communication," in proc. 2005 NSTI Nanotechnology Conference, 2005.

[13] Höfer, H., L. Venance, and C. Giaume, "Control and plasticity of intercellular calcium waves in astrocytes: a modeling approach," Journal of Neuroscience, 22(12), pp 4850-4859, 2002. 
[14] Keener, J. P., "Propagation and its failure in coupled systems of discrete excitable cells," SIAM Journal on Applied Mathematics Volume, vol. 47, Issue 3, pp. 556 - 572, 1987.

[15] Kinosita, K., Jr., R. Yasuda, H. Noji, and K. Adachi, “A rotary molecular motor that can work at near $100 \%$ efficiency," Philos. Trans. R. Soc. Lond. B Biol. Sci., 355:473-489, 2000.

[16] Kitano, H., "Biological robustness," Nature Review Genetics, 5, 826-837, 2004.

[17] Kraus, M., B. Wolf, and B. Wolf, "Crosstalk between cellular morphology and calcium oscillation patterns," Cell Calcium, 19(6), pp. 461-472, 1996.

[18] Krutovskikh, V. A., C. Piccoli and H Yamasaki, "Gap junction intercellular communication propagates cell death in cancerous cells," Oncogene (2002) 21, 1989-1999.

[19] Laer, L., Kloppstech M., Schofl C., Sejnowski T.J., Brabant G., Prank K, "Noise enhanced hormonal signal transduction through intracellular calcium oscillations," Biophysical Chemistry, vol. 91, pp.157-166, 2001.

[20] Liu, J. Q. and T. Nakano, "An information theoretic model of molecular communication based on cellular signalng," in proc. Workshop on Computing and Communications from Biological Systems: Theory and Applications, 2007.

[21] Moore., M., A. Enomoto, T. Nakano, R. Egashira, ,T. Suda, A. Kayasuga, H. Kojima, H. Sakakibara and K. Oiwa, "A design of a molecular communication system for nanomachines using molecular motors," in proc. IEEE Conference on Pervasive Computing and Communications, 2006.

[22] Nakano, T., T. Suda, M. Moore, R. Egashira, A. Enomoto, and K. Arima, "Molecular communication for nanomachines using intercellular calcium signaling", in proc. 5th IEEE conference on nanotechnology, 2005.

[23] T. Nakano, Y. H. Hsu, W. C. Tang, T. Suda, D. Lin, T. Koujin, T. Haraguchi, and Y. Hiraoka, "Microplatform for intercellular communication," in proc. Third Annual IEEE International Conference on Nano/Micro Engineered and Molecular Systems, Jan. 2008.

[24] Niessen, H., H. Harz, P. Bedner, K. Kramer, and K. Willecke, "Selective permeability of different conneixin channels to the second messenger inositol 1,4,5-triphosphate," Journal of Cell Science, 113:1365-1372, 2000.

[25] Prank, K., F. Gabbiani, G. Brabant, "Coding efficiently and information rates in transmembrane signaling," BioSystems vol. 55, pp. 15-22, 2000.

[26] Peracchia, C., Gap junctions: molecular basis of cell communication in health and diseases, Academic Press, 2000 .

[27] Sneyd, J., A. C. Charles and M. J. Sanderson, "A model for the propagation of intercellular calcium waves," Cell Physiology, vol. 266, issue 1 C293-C302, 1994.

[28] Sung, Y. J. , Z. Sung, C. L. Ho, M. T. Lin, J. S. Wang, S. C. Yang, Y. J. Chen, C. H. Lin, "Intercellular calcium waves mediate preferential cell growth toward the wound edge in polarized hepatic cells," Exp Cell Res. 2003 Jul 15;287(2):209-18.

[29] Tanenbaum, A., Computer networks, Prentice Hall, 2003.

[30] Thomas, P.J., D. J. Spencer, S. K. Hampton, P. Park, and J. P. Zurkus, "The diffusion mediated biochemical signal relay channel," in Proc. 17th Annual Conference on Neural Information Processing Systems (NIPS '03), 2003.

[31] Yang, G.-Z. (ed.), Body sensor networks, Springer, 2006.

[32] You, L., R. S. C. Ill, R. Weiss, F. H. Arnold, "Programmed population control by cell-cell communication and regulated killing," Nature, 22 2004. Vol. 428, 868-871.

[33] Weiss, R., S. Basu, S. Hooshangi, A. Kalmbach, D. Karig, R. Mehreja, I. Netravali, "Genetic circuit building blocks for cellular computation, communications, and signal processing," Natural Computing, 2: 47-84, 2003. 